\title{
Measurements of Single Top-Quark Production Using the ATLAS Detector at the LHC
}

\author{
Sebastian Mergelmeyer*, on behalf of the ATLAS Collaboration \\ Humboldt-Universität zu Berlin (DE) \\ E-mail: sebastian.mergelmeyer@cern.ch
}

\begin{abstract}
Recent measurements of the production of single top quarks in proton-proton collisions at a centre-of-mass energy of $13 \mathrm{TeV}$ are presented. The analysed data with an integrated luminosity of $36 \mathrm{fb}^{-1}$ was recorded using the ATLAS detector at the LHC. Differential cross sections for $t W$ production were measured, an analysis of the quantum interference between $t W$ and $t \bar{t}$ production was performed, and evidence for $t Z$ production was found. The results were compared with state-of-the-art theoretical calculations, and no significant deviations from the Standard Model expectations were observed.
\end{abstract}

Sixth Annual Conference on Large Hadron Collider Physics (LHCP2018)

4-9 June 2018

Bologna, Italy

\footnotetext{
* Speaker.
} 


\section{Introduction}

Single top quarks are an important tool to test the predictions of the Standard Model (SM) of particle physics and constrain its parameters. Precision measurements of single top-quark production are also gaining importance as constraints on models of physics beyond the SM, given the lack of clear evidence from direct searches. The production can proceed via the electroweak interaction in several ways, each of which is sensitive to different aspects or potential modifications of the SM. All have in common that they are sensitive to the CKM matrix element $V_{t b}$ as well as modifications of the $t-W-b$ vertex.

The ATLAS collaboration studied the various production modes of single top quarks in $p p$ collisions at centre-of-mass energies of $\sqrt{s}=7,8,13 \mathrm{TeV}$. Evidence for the production involving the $s$-channel exchange of a $W$ boson, previously observed only at the Tevatron, was found [2]. A variety of measurements were performed for the $t$-channel, such as differential cross sections at $8 \mathrm{TeV}$ [3] or fiducial cross sections at $13 \mathrm{TeV}$ [4]. Differential cross sections were also measured for the production in association with a $W$ boson in $t W$ [5] as well as in $t W b$ [6] final states. With the increase in $\sqrt{s}$ and the luminosity, $\mathcal{L}$, it became possible to find evidence for the $t$-channel process with the radiation of an extra $Z$ boson [7]. These proceedings review the latter three analyses, all of which were carried out using $\mathcal{L}=36.1 \mathrm{fb}^{-1}$ of $p p$ collision data at $13 \mathrm{TeV}$ collected with the ATLAS detector [1] in the years 2015 and 2016.

The analyses selected isolated prompt electrons and muons, $\ell=e, \mu^{1}$, as well as jets in the pseudorapidity range of $|\eta|<2.5$ unless noted otherwise. While tau leptons were not explicitly reconstructed, their leptonic decays are included through the $e / \mu$ selection. The jets were defined using the anti- $k_{t}$ algorithm with a distance parameter of 0.4. Jets originating from $b$ quarks were identified based on the characteristics of the decays of $b$-hadrons. Prompt neutrinos, $v$, were collectively reconstructed from the missing transverse momentum, $E_{\mathrm{T}}^{\text {miss }}$. The transverse mass, $m_{\mathrm{T}}$, of a set of objects with indices $K$, can be expressed as: $m_{\mathrm{T}, K}^{2}=\left(\sum_{k \in K} p_{\mathrm{T}, k}\right)^{2}-\left(\sum_{k \in K} p_{x, k}\right)^{2}-\left(\sum_{k \in K} p_{y, k}\right)^{2}$, where $p_{\mathrm{T}}$ denotes the transverse momentum, and $p_{x}\left(p_{y}\right)$ its component along the $x(y)$ axis. Particle level is defined as the state before the particles produced in a collision interact with the detector.

\section{2. $t W$ differential cross sections}

Differential cross sections for the $t W$ production were measured in events with exactly two ${ }^{2}$ oppositely charged leptons. The leptons were required to have $p_{\mathrm{T}}\left(\ell_{1}\right)>27 \mathrm{GeV}$ and $p_{\mathrm{T}}\left(\ell_{2}\right)>$ $20 \mathrm{GeV}$. Events containing one $b$-quark jet and no other jet with $p_{\mathrm{T}}>30 \mathrm{GeV}$ were selected. $Z+$ jets (production of a $Z$ boson in association with jets) events were suppressed using cuts on the combination of the lepton flavours, $m(\ell \ell)$ and $E_{\mathrm{T}}^{\text {miss }}$, leaving $t \bar{t}$ (top-quark pair) production as the only relevant background. This background is particularly challenging because it is nearly indistinguishable from $t W$ production when one of the two $b$-quark jets is not detected, and also has an about 10 times larger cross section.

\footnotetext{
${ }^{1}$ Indices are assigned such that $p_{\mathrm{T}}\left(\ell_{1}\right) \geq p_{\mathrm{T}}\left(\ell_{2}\right)$.

${ }^{2}$ The 2-lepton channel has the advantage that both $W$ bosons are identified in a reliable way, removing a considerable amount of misreconstructed $t \bar{t}$ events as well as almost all non- $t \bar{t}$ background.
} 
In order to separate the signal from $t \bar{t}$ production, a discriminant based on Boosted Decision Trees (BDTs) was constructed from variables such as the transverse momentum of the $t W$ system, $p_{\mathrm{T}}(\ell \ell v v b)$. In the lowest-order diagrams for $t \bar{t}$ and $t W^{-} \bar{b}$ production, it can be seen that this particular variable acts as a proxy for the $p_{\mathrm{T}}$ of the undetected $b$-quark jet. A cut was imposed on the resulting BDT discriminant, $O_{\mathrm{BDT}}>0.3$, increasing the signal-to-background ratio from $1: 5$ to $1: 2$, and reducing the effects of uncertainties in the $t \bar{t}$ modelling.

After subtracting the background estimate, the distributions of the data in six different observables, listed in Table 1, were unfolded to particle level. Distributions for two of the observables are shown in Fig. 1. An iterative method was used for the unfolding, with a number of iterations chosen as to minimise the statistical uncertainties as well as differences between the true and the unfolded distributions. The largest sources of uncertainties were the $t W$ modelling (e.g. choice of generators for the hard scatter and the parton shower), the $t \bar{t}$ modelling, and in some bins the data population. Normalising the differential cross sections was very effective at reducing effects from $t \bar{t}$ modelling.

All studied generators model the data generally well, with a trend to softer spectra in all observables, especially $E(\ell \ell b)$. PowHEG-Box+Herwig++ was found to deviate more than the alternatives from the data in certain bins of the differential cross sections. The differences between the DR and the DS scheme ${ }^{3}$ as well as between the radHi and radLo tunings (of radiation and theory scale) were negligible.

\section{Quantum interference between $t W$ and $t \bar{t}$ production}

At next-to-leading order (NLO) in QCD, diagrams such as $g g \rightarrow t W^{-} \bar{b}$ appear in the perturbative expansion for $t W$ production. These lead to a $W^{+} W^{-} b \bar{b}$ final state after the decay of the top quarks, just like $t \bar{t}$ production, giving rise to interference. Some of the diagrams even look like $t \bar{t}$ production followed by the decay $\bar{t} \rightarrow W^{-} \bar{b}$, and their double-counting as both $t W$ and $t \bar{t}$ has to be avoided in one way or another [8]. An excellent overview of the available options, namely diagram removal (DR), diagram subtraction (DS), and diagram removal 2 (DR2) is given in Ref. [9].

\begin{tabular}{lcccccc}
\hline Observable & $E(b)$ & $m\left(\ell_{1} b\right)$ & $m\left(\ell_{2} b\right)$ & $E(\ell \ell b)$ & $m_{\mathrm{T}}(\ell \ell v v b)$ & $m(\ell \ell b)$ \\
Degrees of freedom & 4 & 5 & 3 & 5 & 3 & 5 \\
\hline Powheg+PythiA6 DR & 0.31 & 0.34 & 0.45 & 0.15 & 0.56 & 0.55 \\
PowHEG+Pythia6 DS & 0.29 & 0.30 & 0.46 & 0.11 & 0.49 & 0.50 \\
aMC@ NLO+Herwig++ & 0.23 & 0.37 & 0.49 & 0.12 & 0.61 & 0.61 \\
PowHEG+Herwig++ & 0.18 & 0.15 & 0.52 & 0.05 & 0.57 & 0.40 \\
PowHEG+PythiA6 radHi & 0.30 & 0.38 & 0.48 & 0.16 & 0.60 & 0.60 \\
Powheg+PythiA6 radLo & 0.29 & 0.33 & 0.45 & 0.14 & 0.56 & 0.55 \\
\hline
\end{tabular}

Table 1: $p$-values from the comparison of the normalised differential $t W$ cross sections to several Monte Carlo predictions. [5]

\footnotetext{
${ }^{3}$ See Section 3, but note the analysis here was designed to be insensitive to the choice of scheme, by selecting events with exactly 1 jet, and using a mixture of DR and DS $t W$ events in the BDT training.
} 

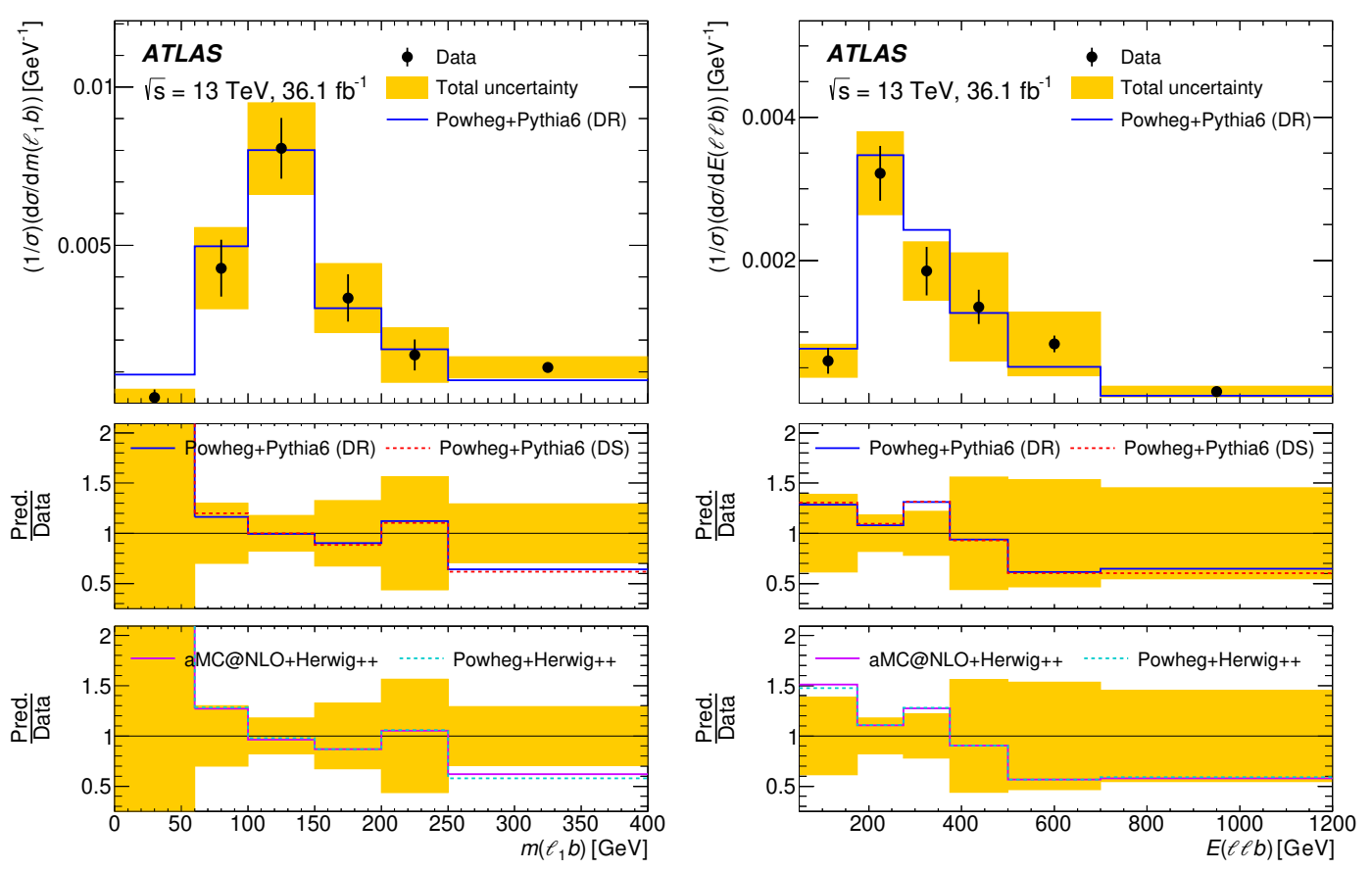

Figure 1: Normalised differential cross sections for $t W$ production as functions of $m\left(\ell_{1} b\right)$ (left) and $E(\ell \ell b)$ (right). The measured cross sections, shown as markers, are compared to several Monte Carlo models. The bars through the markers indicate the statistical, the yellow band the total uncertainty. [5]

The choice generally plays a minor role in measurements of single top-quark production, but is associated with sizable uncertainties in the $t W$ modelling elsewhere, for example in searches for top squarks in the context of supersymmetry. A radically different and technically challenging approach is to calculate the $W^{+} W^{-} b \bar{b}$ final state at NLO QCD, avoiding the distinction between $t W$ and $t \bar{t}$. In particular PowHEG-Box-Res is able to simulate $e^{ \pm} v \mu^{ \pm} \bar{v} b \bar{b}$ events, which can then be extrapolated to all flavor combinations including fully leptonic tau decays.

The analysis discussed here constitutes a unique benchmark for generators of $t W$ events, especially their treatment of the $t W-t \bar{t}$ overlap. The basic selection is similar to that in Section 2; however, events with 2 jets are picked in order to enhance the interference. Note that if a $W b$ pair does not originate from the decay of an on-shell top-quark, $\max \left(m\left(W^{+} b\right), m\left(W^{-} \bar{b}\right)\right)$ may exceed the top-quark mass, $m_{t}$. Taking this and the limitations of neutrino and jet-charge reconstruction into account, the following observable was constructed:

$$
\begin{aligned}
& m_{b \ell}^{\operatorname{minimax}}=\min \{ \max \left\{m\left(\ell_{1} b_{1}\right), m\left(\ell_{2} b_{2}\right)\right\}, \\
&\left.\max \left\{m\left(\ell_{2} b_{1}\right), m\left(\ell_{1} b_{2}\right)\right\}\right\} .
\end{aligned}
$$

After subtracting the backgrounds, primarily $t \bar{t}$ production in association with $b$-quark jets, the observed distribution of $m_{b \ell}^{\operatorname{minimax}}$ was unfolded to particle level. Figure 2 shows the raw and the unfolded distributions. A very good description of the full distribution by PowHEg-Box-Res can be observed. The predictions based on the DR and the DS schemes start to diverge above $m_{t}$, but 

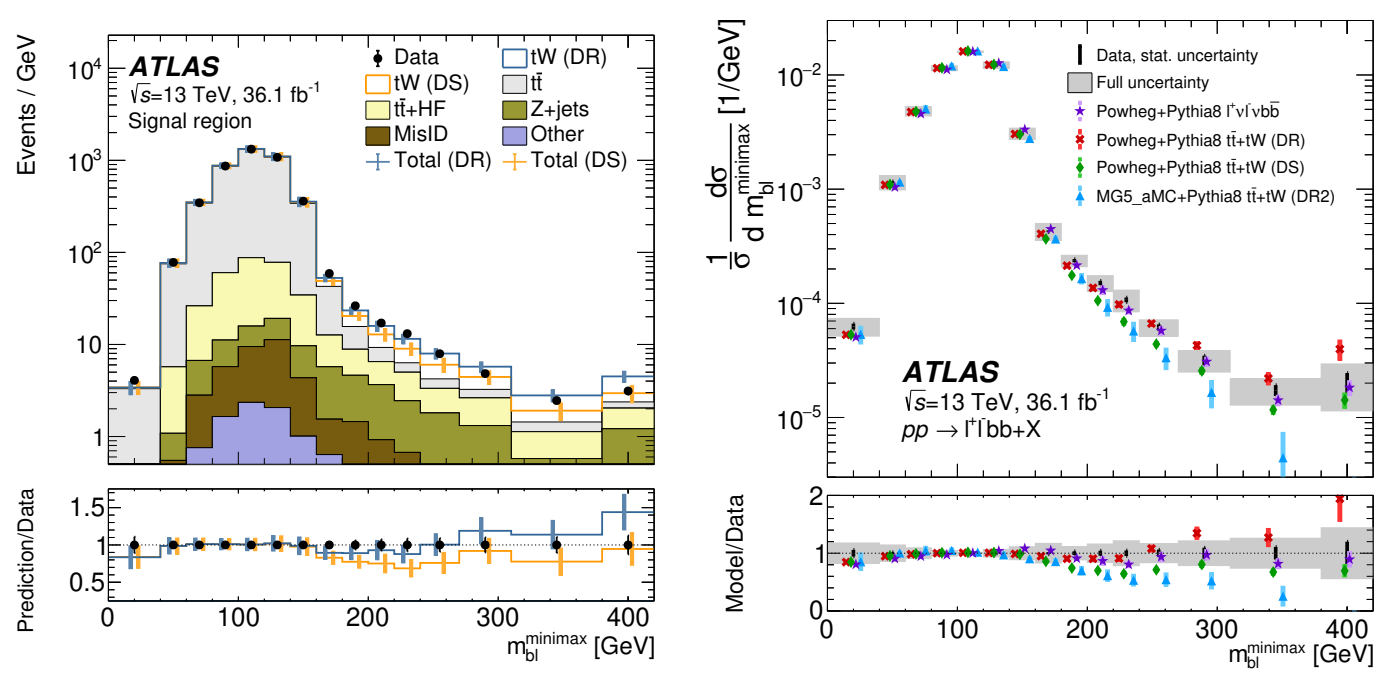

Figure 2: Distribution and differential cross section for $t W+t \bar{t}$ production as a function of $m_{b \ell}^{\operatorname{minimax}}$. The observed data are compared to selected MC models with different treatment of the $t W-t \bar{t}$ overlap. The black error bars correspond to the statistical error of the data. On the left, the colored bars include all systematic uncertainties in the prediction. On the right, the colored bars indicate the uncertainty of the theory models, including PDF and scale variations. [6]

stay less than 2 standard deviations away from the data. Note that the detector acceptances for $t W$ events in the DR and the DS schemes are slightly different, which was taken into account in the systematic uncertainty; the difference is small compared to the deviations seen in Fig. 2 (right). The DR2 scheme significantly underestimates the tail of the distribution ${ }^{4}$.

\section{Evidence for $t Z j$ production}

The $t$-channel production of single top quarks in association with a $Z$ boson was measured for the first time [7]. The additional $Z$ boson not only adds sensitivity to the $t-t-Z$ but also to the $W-W-Z$ coupling. The measurement was performed in final states with 3 charged leptons, using staggered $p_{\mathrm{T}}$ cuts at 15,25 and $28 \mathrm{GeV}$. An opposite-charge same-flavour pair of leptons was required, satisfying $m(\ell \ell)=91 \pm 10 \mathrm{GeV}$. The remaining lepton, $\ell_{W}$, was used in the cut $m_{\mathrm{T}}\left(\ell_{W} E_{\mathrm{T}}^{\text {miss }}\right)>20 \mathrm{GeV}$ in order to suppress backgrounds without a $W$ boson. Two jets with $p_{\mathrm{T}}>$ $30 \mathrm{GeV}$ were required, one $b$-tagged jet and one forward jet, which is characteristic for $t$-channel events. The forward jet was looked for in an extended pseudorapidity range $|\eta|<4$.5. Using 10 variables such as the reconstructed mass of the top-quark candidate and the pseudorapidity of the forward jet, a discriminant based on a neural network was constructed (Fig. 3).

The signal process was calculated at leading order (LO) in QCD using the narrow-width approximation for the top quark and the $Z$ boson. Backgrounds containing a fake lepton, almost exclusively $t \bar{t}$ and $Z+$ jets, were estimated using a combination of simulation and data-driven meth-

\footnotetext{
${ }^{4}$ A different generator, aMC@NLO, was used for the DR2 prediction. aMC@NLO and PowHEG-Box yield compatible DR predictions, as can be seen in the auxiliary material of Ref. [6].
} 

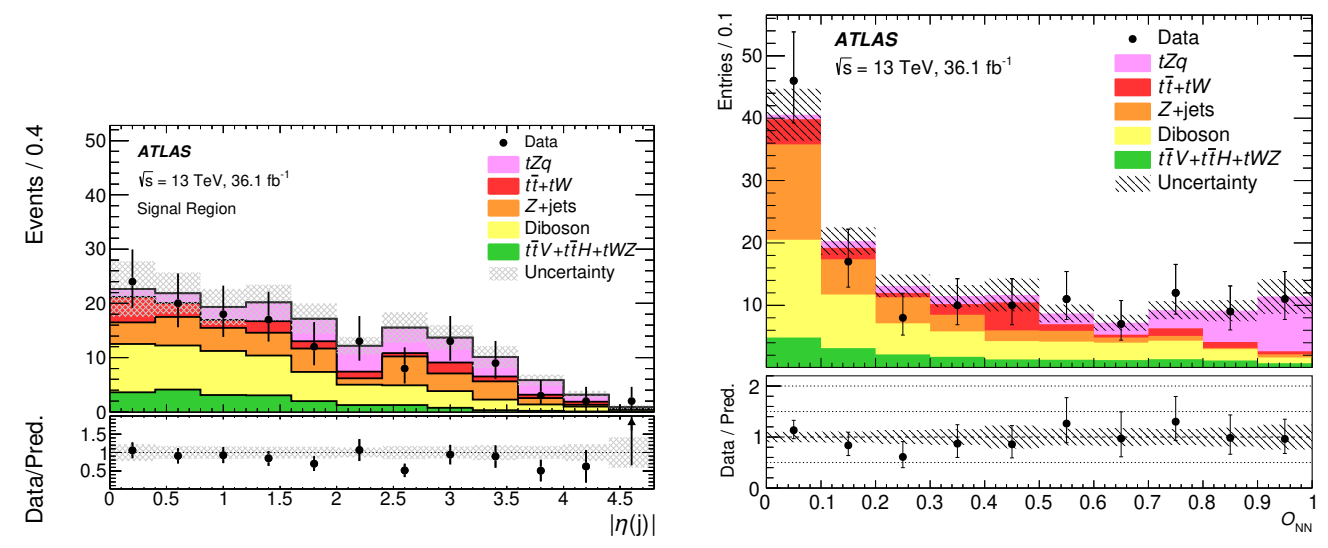

Figure 3: Distributions of the pseudorapidity of the forward jet (left) and the neural network output (right) in the analysis of $t Z j$ production. [7]

ods. Diboson (predominantly $W Z+$ jets) and $t \bar{t} V$ events were simulated at LO QCD, and normalised using a control region and NLO predictions, respectively.

The signal and background expectations were fitted to the observed data, using a free parameter for the normalisation of the signal as well as multiple nuisance parameters for the systematic uncertainties. After (before) the fit, $143 \pm 11(163 \pm 12)$ events were expected, $26 \pm 8(35 \pm 9)$ of which were attributed to the $t Z j$ signal. The measured cross section is:

$$
\sigma=600 \pm 170 \text { (stat.) } \pm 140 \text { (syst.) fb at } \sqrt{s}=13 \mathrm{TeV},
$$

where the systematic uncertainties are mainly due to the tuning of radiation and renormalisation scale, and the amount of diboson background. The cross section is consistent with the calculation at NLO QCD obtained through aMC@NLO, 800 fb, which comes with an about 7\% uncertainty due to the choice of scales and PDFs. The background-only hypothesis was rejected at a significance level of 4.2 standard deviations (for 5.4 expected), providing clear evidence for the existence of the $t Z j$ process.

\section{References}

[1] ATLAS Collaboration, The ATLAS Experiment at the CERN Large Hadron Collider, JINST 3 (2008) S08003

[2] ATLAS Collaboration, Evidence for single top-quark production in the s-channel in proton-proton collisions at $\sqrt{s}=8 \mathrm{TeV}$ with the ATLAS detector using the Matrix Element Method, Phys. Lett. B 756 (2016) 228 [arXiv: 1511.05980]

[3] ATLAS Collaboration, Fiducial, total and differential cross-section measurements of t-channel single top-quark production in pp collisions at $8 \mathrm{TeV}$ using data collected by the ATLAS detector, Eur. Phys. J. C 77 (2017) 531 [arXiv: 1702.02859]

[4] ATLAS Collaboration, Measurement of the inclusive cross-sections of single top-quark and top-antiquark t-channel production in pp collisions at $\sqrt{s}=13 \mathrm{TeV}$ with the ATLAS detector, JHEP 04 (2017) 86 [arXiv: 1609.03920] 
[5] ATLAS Collaboration, Measurement of differential cross-sections of a single top quark produced in association with a W boson at $\sqrt{s}=13 \mathrm{TeV}$ with ATLAS, Eur. Phys. J. C 78 (2018) 186 [arXiv: 1712.01602$]$

[6] ATLAS Collaboration, Probing the quantum interference between singly and doubly resonant top-quark production in pp collisions at $\sqrt{s}=13 \mathrm{TeV}$ with the ATLAS detector, submitted to Phys. Rev. Lett. [arXiv: 1806.04667]

[7] ATLAS Collaboration, Measurement of the production cross-section of a single top quark in association with a $Z$ boson in proton-proton collisions at $13 \mathrm{TeV}$ with the ATLAS detector, Phys. Lett. B 780 (2018) 557 [arXiv: 1710.03659]

[8] E. Re, Single-top Wt-channel production matched with parton showers using the PowHEG method, Eur. Phys. J. C 71 (2011) 1547 [arXiv: 1009. 2450]

[9] F. Demartin et al., tWH associated production at the LHC, Eur. Phys. J. C 77 (2017) 34 [arXiv: 1607.05862] 